F.A.S.T. is an easy way to remember the sudden signs of a stroke.
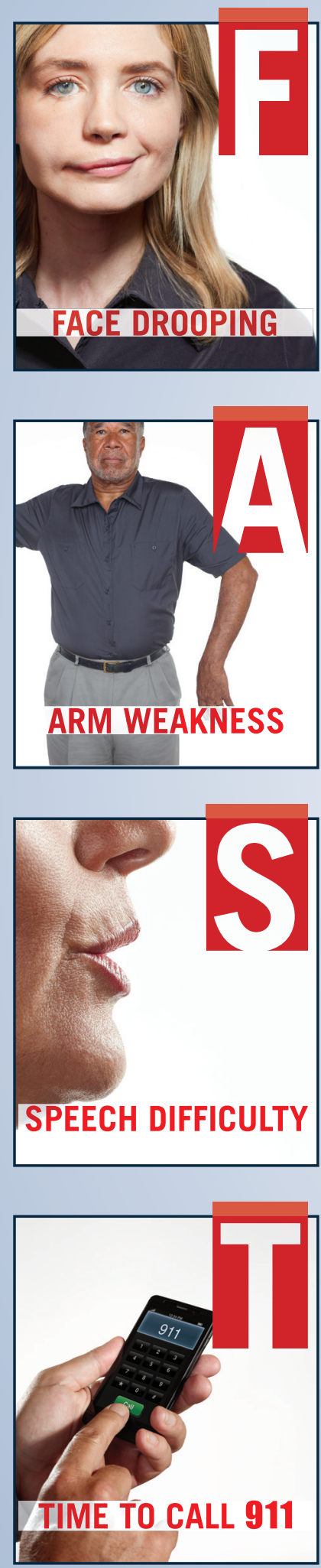

\section{Face Drooping}

Does one side of the face droop or is it numb? Ask the person to smile.

\section{Arm Weakness}

Is one arm weak or numb? Ask the person to raise both arms. Does one arm drift downward?
Beyond F.A.S.T. Other Symptoms you should know

- Sudden numbness or weakness of the leg

- Sudden confusion or trouble understanding

- Sudden trouble seeing in one or both eyes

- Sudden trouble walking, dizziness, loss of balance or coordination

- Sudden severe headache with no known cause

\section{Speech Difficulty}

Is speech slurred, are they unable to speak, or are they hard to understand? Ask the person to repeat a simple sentence, like "the sky is blue." Is the sentence repeated correctly?
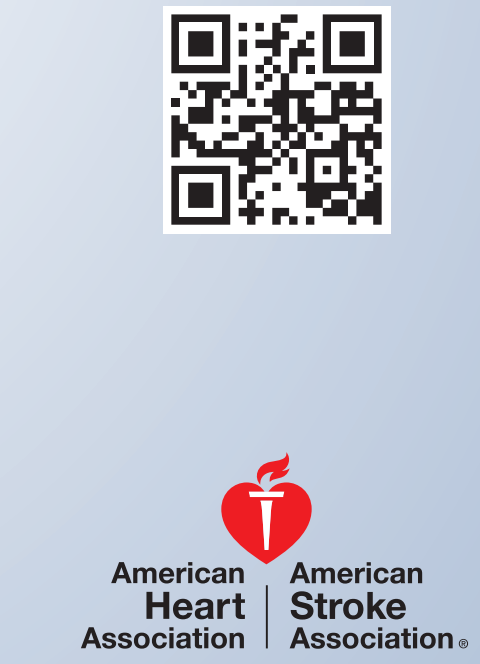

Together to End Stroke ${ }^{\text {Tm }}$

Time to call 9-1-1

If the person shows any of these symptoms, even if the symptoms go away, call 9-1-1 and get them to the hospital immediately. 